\title{
Growth and investigation of m-plane (In)GaN buffer layers on $\mathrm{LiAlO}_{2}$ substrates
}

C. Mauder ${ }^{1}$, L. Rahimzadeh Khoshroo ${ }^{1}$, H. Behmenburg ${ }^{1}$, T. C. Wen ${ }^{1}$, Y. Dikme ${ }^{2}$, M. V. Rzheutskii ${ }^{3}$, E. V. Lutsenko ${ }^{3}$, G. P. Yablonskii ${ }^{3}$, A. Kharchenko ${ }^{4}$, J. F. Woitok ${ }^{4}$, M. M. C. Chou ${ }^{5}$, M. Heuken ${ }^{1,2}$, H. Kalisch ${ }^{1}$, and R. H. Jansen ${ }^{1}$

${ }^{1}$ Chair of Electromagnetic Theory, RWTH Aachen University, Kopernikusstr. 16, 52074 Aachen, Germany

${ }^{2}$ AIXTRON AG, Kackertstr. 15-17, 52072 Aachen, Germany

${ }^{3}$ Stepanov Institute of Physics, National Academy of Sciences of Belarus, Independence Ave, 68, Minsk 220072, Belarus

${ }^{4}$ PANalytical B.V., P.O. Box 13, 7600 AA Almelo, The Netherlands

${ }^{5}$ Department of Material Science \& Opto-Electronic Engineering, National Sun Yat-Sen University, Taiwan, R.O.C.

PACS 81.15.Gh Chemical vapour deposition, 81.05.Ea III-V semiconductors, 61.10.Nz X-ray diffraction, 78.55.Cr Photoluminescence

We deposited pure m-plane GaN (1-100) layers on $\mathrm{LiAlO}_{2}$ (100) substrates by MOVPE using Mg-doped InGaN buffer layers of various thickness. These sealing layers are grown under nitrogen ambient and help to improve the film coalescence while reducing the oxygen background doping of the GaN films. We show that for an increasing thickness of the InGaN:Mg buffer layer, the highly strained GaN layer slightly relaxes. An improved surface morphology can be achieved for thicker InGaN:Mg buffers while the defect density clearly increases above a certain thickness as it can be seen in X-ray rocking curve measurements. In temperature-dependent photoluminescence spectroscopy, we observe not only peaks due to DAP transitions at an energy of around $3.3 \mathrm{eV}$, but also a defect related peak at $3.43 \mathrm{eV}$ as well as a peak at $3.49 \mathrm{eV}$ connected to free and bound excitonic transitions. It is interesting to note that for an increasing thickness of the InGaN:Mg buffer, we detect a higher intensity of the excitonic transition while the defect-related signal seen at $3.43 \mathrm{eV}$ is getting more pronounced. It might be possible that during the film relaxation, basal plane stacking faults are introduced into the layer.

Pre-print version of journal article. Original version available online (www.elsevier.com/locate/jcrysgro)

C. Mauder et al., J. Cryst. Growth 310 (2008) 4976

DOI:10.1016/j.jcrysgro.2008.07.053

\section{Introduction}

The increasing demand for highly efficient nitride blue and green light emitting diodes (LEDs) has attracted great interest for the growth of nonpolar GaN. In contrast to the common growth of nitride material in the polar c-axis, the absence of polarization-induced electric fields in the growth direction for nonpolar structures leads to a better overlap of electron and hole wave functions in quantum wells and therefore to a more efficient carrier recombination [1]. As a second advantage, nonpolar light emitters exhibit a much lower wavelength shift with higher driving currents due to the absence of polarization field screening normally occurring in c-plane GaN [2]. One interesting approach to achieve this is to grow on (100) $\mathrm{LiAlO}_{2}$ substrates, which enables a deposition of $\mathrm{GaN}$ in m-plane orientation with a rather small substrate-lattice mismatch of only $1.7 \%$ and $0.3 \%$ in [11-20] and [0001] direction of GaN, respectively [3]. The substrates can be manufactured by conventional Czochralski pulling method which allows potentially cheap large-size wafer production. One of the challenges with this material is the sensitivity to hydrogen and water. On the other hand, this offers a possibility of an easy substrate removal after device processing for an improved light extraction and heat management.

In first literature reports, mainly molecular beam epitaxy (MBE) was used to deposit pure m-plane GaN on $\mathrm{LiAlO}_{2}[1,3,4]$ but also successful growth by hydride vapor phase epitaxy (HVPE) has been reported [5]. The challenges with the growth by metal organic vapor phase epitaxy (MOVPE) on this substrate are related to the demand for high growth temperatures and hydrogen ambient to achieve high-quality material, both of which can cause serious substrate damage. Nevertheless, several groups succeeded in growing $\mathrm{GaN}$ on $\mathrm{LiAlO}_{2}$ by MOVPE [6,7]. But the layers still exhibit either a low phase purity, rough surfaces or a high background carrier concentration, the latter being caused by an outdiffusion of oxygen from the substrate at elevated temperatures. As we have shown in an earlier report, the introduction of a thin layer of Mg-doped InGaN helps to effectively seal the substrate surface and to block the outdiffusion of oxygen into the layer [8]. By using this approach of growing m-plane GaN, nonpolar MQW structures with high wavelength stability could be produced [9].

To study and improve this method, we investigated m-plane GaN buffers using InGaN:Mg sealing layers of different thicknesses and their influence on the defect characteristics of the GaN films. 


\section{Experimental}

The growth experiments were performed in single-wafer, horizontal-flow AIXTRON MOVPE reactors equipped with TMGa, TMIn and $\mathrm{Cp}_{2} \mathrm{Mg}$ as precursors. Polished (100) $\mathrm{LiAlO}_{2}$ substrates with an average substrate root mean square (RMS) roughness of $0.4-0.5 \mathrm{~nm}$ were used without any cleaning procedure. More details about their fabrication can be found elsewhere [10]. The process was initiated with an in-situ nitridation by exposing the wafer to $\mathrm{NH} 3$ at $1050^{\circ} \mathrm{C}$. The Mg-doped InGaN sealing layer with a thickness between 25 and $150 \mathrm{~nm}$ was deposited at $900^{\circ} \mathrm{C}$. This was followed by a $200 \mathrm{~nm}$ thick Mg-doped $\mathrm{GaN}$ film at $1000^{\circ} \mathrm{C}$ and a $500 \mathrm{~nm}$ thick undoped GaN layer grown at $1050^{\circ} \mathrm{C}$. Phase purity and crystal quality of the m-plane GaN were attested by high resolution X-ray diffraction (HRXRD) using a PANalytical Material Research Diffractometer and software analysis tool. The evaluation of strain and indium fraction was performed using methods and elastic constants given in [11]. Atomic force microscopy (AFM) scans were performed to measure the surface roughness. To determine optical properties, we carried out temperature-dependent photoluminescence spectroscopy (PL) using a He closed-cycle cryostat and a $325 \mathrm{~nm} \mathrm{HeCd}$ Laser with an excitation power density of $\sim 1 \mathrm{~W} / \mathrm{cm}^{2}$. Hall measurements were conducted in van der Pauw geometry.

\section{Results and discussion}

To prevent damaging of the sensitive $\mathrm{LiAlO}_{2}$ surface, we used a relatively low reactor temperature and nitrogen ambient in the first growth step. As a starting layer, we chose Mg-doped InGaN since this material can be grown under nitrogen ambient at reasonable quality. The Mg doping serves the purpose to block oxygen from diffusing out of the substrate which will otherwise lead to a very high n-type background carrier concentration in the GaN layer $[8,12]$. In Fig. 1, HRXRD $2 \Theta / \omega$ scans in triple axis geometry of four $\mathrm{InGaN}: \mathrm{Mg} / \mathrm{GaN}$ structures having various InGaN:Mg buffer thicknesses are displayed. Beside of the substrate peak at $34.69^{\circ}$, we can observe peaks for m-plane $\mathrm{GaN}$ and $\mathrm{m}$-plane $\mathrm{InGaN}$ while no c-plane material is detected. With rising $\mathrm{InGaN}: \mathrm{Mg}$ thickness, we see an increasing intensity of the correlated peak. The peaks for $\mathrm{GaN}$ are located around $32.27^{\circ}$, which is similar to what other groups report $[1,3]$. But for the thickest InGaN:Mg buffer, this peak shifts to $32.32^{\circ}$ which is closer to its ideal position at $32.39^{\circ}$. This can be explained by a relaxation of the strained $\mathrm{GaN}$ film, possibly supported by the underlying InGaN:Mg layer. Reciprocal space maps (not shown here) around (2-200), (2-310) and (2-20-1) reflections on the sample with $25 \mathrm{~nm} \mathrm{InGaN:Mg}$ revealed an In molar fraction of $3.4 \%$ and an in-plane compressive strain for the GaN layer of $-1.1 \%$ and $-0.1 \%$ in $[11-20]$ and [0001] direction, respectively. These values are consistent with the lattice mismatch and other reports about MBE-grown m-plane GaN layers on $\mathrm{LiAlO}_{2}[4,13]$. In contrast to that, the sample with the thickest InGaN:Mg layer features an In fraction of $7.6 \%$ and strain values of $-0.5 \%$ and $0.1 \%$ for the [11-20] and [0001] direction, respectively, indicating a relaxation of the GaN film. The InGaN:Mg film is also strained and exhibits a slight shift to lower angles with rising In content in (2-200) $2 \Theta / \omega$ scans (not shown here). Because of the superposition of the InGaN and GaN (1-100) peaks, the shift cannot be observed in Fig. 1. We ascribe this increase of the In concentration to the well-known indium pulling effect [14].

In order to investigate the influence of the InGaN:Mg layer and its thickness on the GaN material quality, X-ray rocking curve (XRC) measurements with the in-plane component of the incident beam along GaN [0001] and AFM scans for a $5 \times 5 \mu \mathrm{m}^{2}$ area were performed on all samples. The direction of the incident beam was chosen parallel and perpendicular to the c-axis to uncover anisotropic defect influences. In Fig. 2, both the XRC full width at half maximum (FWHM) values and the AFM root mean square (RMS) roughness values are shown. The surface morphology shown in the inset of Fig. 2 for a $25 \mathrm{~nm}$ (a) and $150 \mathrm{~nm}$ (b) thick InGaN:Mg buffer exhibits trenches indicating a not fully coalesced surface. The average surface roughness clearly improves with the thickness of the underlying InGaN:Mg coating which is possibly caused by a better layer coalescence in the first growth steps. Apart from that, we see an increase of the FWHM values parallel to the c-axis above a thickness of $50 \mathrm{~nm}$, while the values measured in perpendicular direction show a slightly oppositional trend. As in the former geometry the measurement should become more sensitive to basal plane stacking faults (BSF) [15], this indicates a possible increase of such defects connected to the strain relaxation for thicker InGaN:Mg films.

To obtain more information on the defect characteristics in our layers, we employed temperature-dependent PL spectroscopy. Fig. 3 shows the PL spectra of a GaN layer with $50 \mathrm{~nm}$ InGaN:Mg taken in the temperature range from 10 to $300 \mathrm{~K}$. For low temperatures, at least two strong peaks can be seen for energies between 3.1 and $3.3 \mathrm{eV}$, which we think might origin from DAP transitions due to the oxygen background concentration and residual $\mathrm{Mg}$ doping $[16,17]$. Furthermore, we observe a high signal at $3.43 \mathrm{eV}$, which has been reported to be the energetic position for stacking faults in $\mathrm{m}$ - and a-plane $\mathrm{GaN}[18,19]$. While this signal is very high for low temperature, it is quenched quickly with temperature increase and disappears from the spectrum at $120 \mathrm{~K}$. Another third peak is visible in Fig. 3 around $3.49 \mathrm{eV}$. We observe a complicated temperature dependence of the position of this peak, namely a high-energy shift for $10-60 \mathrm{~K}$ and a low-energy shift at temperatures higher than $60 \mathrm{~K}$. Such complex behaviour is caused by the superposition of the bound and free excitonic (BE+FE) signal in one broad peak. We can see that the signal from bound excitons determines the energetic peak position for very low temperatures. At temperature enhancement, the peak shifts smoothly from bound to free exciton position which dominates the peak for temperatures higher than $70 \mathrm{~K}$. It is also interesting to note that with increasing thickness of the InGaN:Mg layer, we observe a clear tendency for the intensity ratio of the excitonic and defect- 
related transitions. This trend is illustrated in Fig. 4. While the BSF-related peak at $3.43 \mathrm{eV}$ shows an increasing intensity for thicker InGaN:Mg layers, the signal at $3.49 \mathrm{eV}$ is getting weaker. We think this may be caused by an increase of the stacking fault density in the m-plane GaN layers.

\section{Conclusion}

We have deposited and characterised m-plane GaN films grown by MOVPE using Mg-doped InGaN buffer layers of various thicknesses. It was shown that the thickness of this starting layer affects the properties of the overlying $\mathrm{GaN}$ film. Thicker InGaN:Mg layers lead to a distinct relaxation of the highly strained GaN. Furthermore, thick buffer layers produce an improved surface morphology. However, we also observe a decrease in crystal quality, possibly caused by an increased BSF density. Optical investigations yield also a peak at $3.43 \mathrm{eV}$ which is present in all of our samples and can be ascribed to BSF-related transitions. The higher intensity of this peak for thicker InGaN:Mg layers confirms our statement about the rising defect density. We also find a signal from DAP transitions in our layer, which is due to the still high concentration of oxygen impurities and some residual $\mathrm{Mg}$ doping. Beside of that, another peak at $3.49 \mathrm{eV}$ which is connected to both free and bound excitons is visible already indicating quite sufficient quality of our layers.

\section{Acknowledgments}

We gratefully acknowledge financial support by the Deutsche Forschungsgemeinschaft (DFG). References

\section{References}

[1] P. Waltereit, O. Brandt, M. Ramsteiner, A. Trampert, H. T. Grahn, J. Menninger, M. Reiche, R. Uecker, P. Reiche, and K. H. Ploog, phys. stat. sol. (a) 180, 133 (2000).

[2] A. Chakraborty, B. A. Haskell, S. Keller, J. S. Speck, S. P. DenBaars, S. Nakamura, and U. K. Mishra, Jpn. J. Appl. Phys. 44, 173 (2005).

[3] Y. J. Sun, O. Brandt, U. Jahn, T. Y. Liu, A. Trampert, S. Cronenberg, S. Dhar, and K. H. Ploog, J. Appl. Phys. 92, 5714 (2002).

[4] J. W. Gerlach, A. Hofmann, T. Höche, F. Frost, B. Rauschenbach, and G. Benndorf, Appl. Phys. Lett. 88, 011902 (2006).

[5] B. A. Haskell, A. Chakraborty, F. Wu, H. Sasano, P. T. Fini, S. P. DenBaars, J. S. Speck, and S. Nakamura, J. Elect. Mat. 34, 357 (2005).

[6] K. Xu, J. Xu, P. Deng, R. Qiu, and Z. Fang, phys. stat. sol. (a) 176, 589 (1999).

[7] C. Liu, Z. Xie, P. Han, B. Liu, L. Li, J. Zou, S. Zhou, L. H. Bai, Z. H. Chen, R. Zhang, and Y. Zheng, J. Crystal Growth 298, 228 (2007).

[8] Y. Dikme, P. van Gemmern, B. Chai, D. Hill, A. Szymakowski, H. Kalisch, M. Heuken, and R. H. Jansen, phys. stat. sol. (c) 2, 2161 (2005).

[9] H. Behmenburg, T. C. Wen, Y. Dikme, C. Mauder, L. R. Khoshroo, M. M. C. Chou, M. V. Rzheutskii, E. V. Lutsenko, G. P. Yablonskii, J. Woitok, H. Kalisch, R. H. Jansen, and M. Heuken, phys. stat. sol. (b) 245, 893 (2008).

[10] M. M. C. Chou, S. J. Huang, and C. W. C. Hsu, J. Crystal Growth 303, 585 (2007).

[11] M. Tsuda, H. Furukawa, A. Honshio, M. Iwaya, S. Kamiyama, H. Amano, and I. Akasaki, Jpn. J. Appl. Phys. 45, 2509 (2006).

[12] J. K. Sheu and G. C. Chi, J. Phys.: Condens. Matter 14, 657 (2002).

[13] C. Rivera, J. Appl. Phys. 101, 053527-1 (2007).

[14] K. Hiramatsu, Y. Kawaguchi, M. Shimizu, N. Sawaki, T. Zheleva, R. F. Davis, H. Tsuda, W. Taki, N. Kuwano, and K. Oki, MIJ-NSR 2, 6 (1997).

[15] T. Paskova, R. Kroeger, S. Figge, D. Hommel, V. Darakchieva, B. Monemar, E. Preble, A. Hanser, N. M. Williams, and M. Tutor, Appl. Phys. Lett. 89, 051914-1 (2006).

[16] S. Strite and H. Morokoc, J. Vac. Sci. Technol. 10, 1237 (1992).

[17] C. H. Hong, D. Pavlidis, S. W. Brown, and S. C. Rand, J. Appl. Phys. 77, 1705 (1995).

[18] M. A. Reshchikov, A. Teke, H. P. Maruska, D. W. Hill, and H. Morkoc, Mater. Res. Soc. Sym. Proc. 798, Y5.53.1 (2004).

[19] R. Liu, A. Bell, F. A. Ponce, C. Q. Chen, J. W. Yang, and M. A. Khan, Appl. Phys. Lett. 86, 021908 (2005). 


\section{Figures}

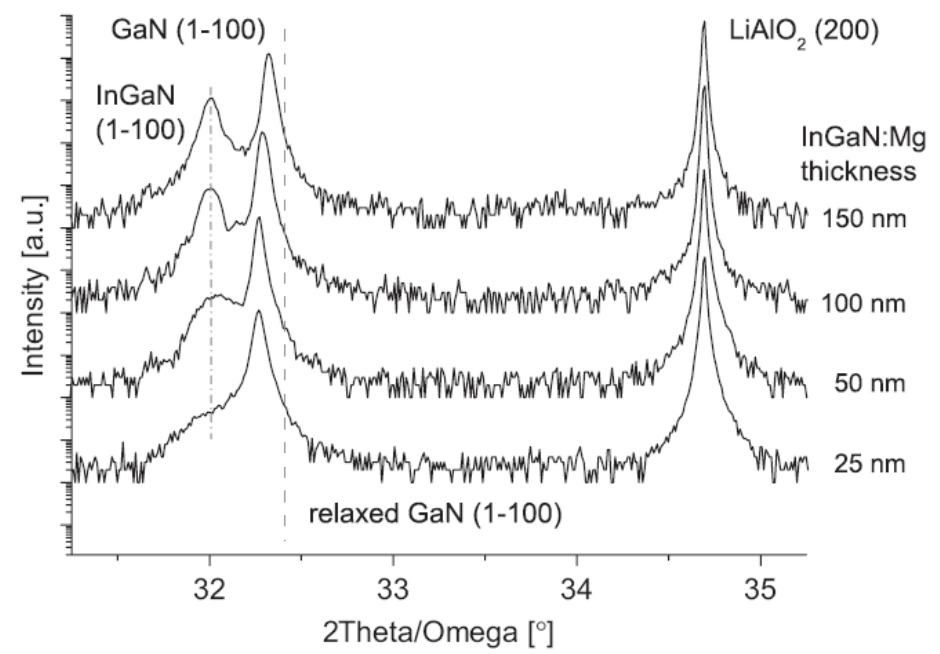

Figure $12 \Theta / \omega$ scans of InGaN:Mg/GaN buffers on $\mathrm{LiAlO}_{2}$ with varying InGaN:Mg thickness. The dotted line on the left side is a guide to the eye. The dashed line shows the unstrained position of m-plane GaN.

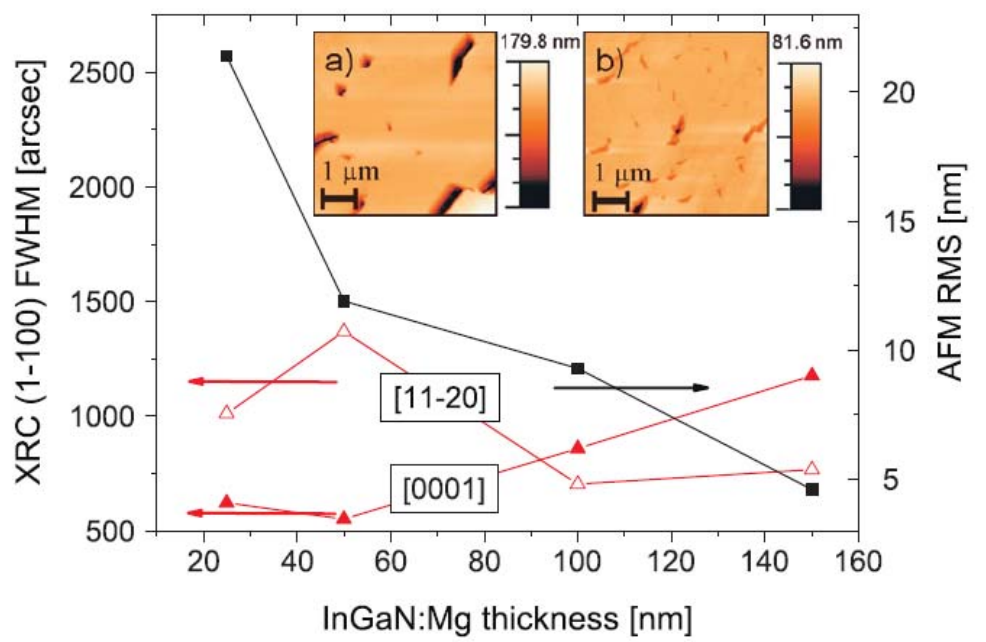

Figure 2 Development of the (1-100) XRC FWHM with the in-plane component of the incident beam parallel ([0001], closed triangles) and perpendicular ([1-120], open triangles) to the c-axis and AFM roughness for various InGaN:Mg buffer layer thicknesses. The inset shows AFM surface scans for the samples with $25 \mathrm{~nm}$ (a) and $150 \mathrm{~nm}$ (b) thick InGaN:Mg buffer.

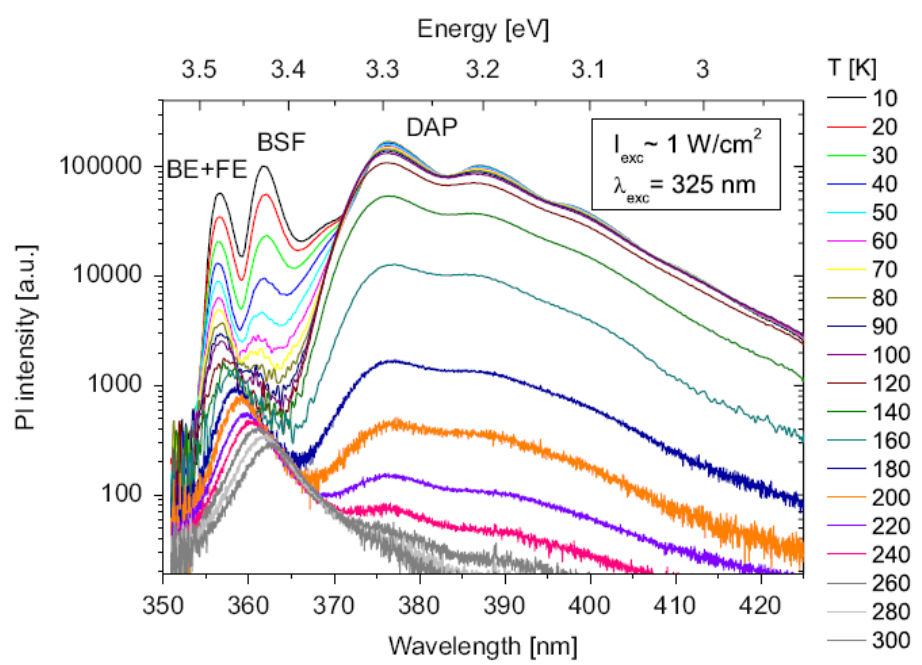

Figure 3 Temperature-dependent PL spectra for an m-plane GaN structure with $50 \mathrm{~nm} \mathrm{InGaN:Mg} \mathrm{buffer.}$ 


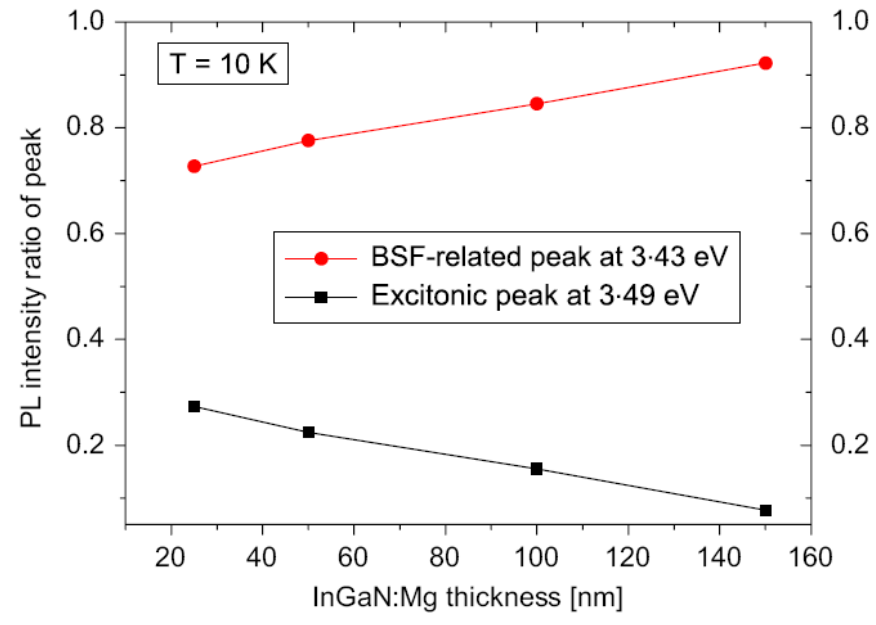

Figure 4 Low-temperature PL signal intensity ratios of both excitonic and defect-related peaks versus InGaN:Mg thickness. 